The Journal of Fourier Analysis and Applications

\title{
Sampling Theorem and Discrete Fourier Transform on the Hyperboloid
}

\author{
M. Calixto, J. Guerrero and J.C. Sánchez-Monreal \\ ABSTRACT. Using Coherent-State (CS) techniques, we prove a sampling theorem for holomorphic \\ functions on the hyperboloid (or its stereographic projection onto the open unit disk $\mathbb{D}_{1}$ ), seen as a \\ homogeneous space of the pseudo-unitary group $S U(1,1)$. We provide a reconstruction formula for \\ bandlimited functions, through a sinc-type kernel, and a discrete Fourier transform from $N$ samples \\ properly chosen. We also study the case of undersampling of band-unlimited functions and the con- \\ ditions under which a partial reconstruction from $N$ samples is still possible and the accuracy of the \\ approximation, which tends to be exact in the limit $N \rightarrow \infty$.
}

\section{Introduction}

In a previous article [1], we proved sampling theorems and provided discrete Fourier transforms for holomorphic functions on the Riemann sphere, using the machinery of Spin CS related to the special unitary group $S U(2)$, which is the double cover of the group $S O(3)$ of motions of the sphere $\mathbb{S}^{2}$. Here we study similar discretization problems for its noncompact counterpart $S O(2,1)$ (the group of motions of the Lobachevsky plane) or, more precisely, for its double cover $S U(1,1)$. Both, $S U(2)$ and $S U(1,1)$, appear as the underlying symmetry groups of many physical systems for which they constitute a powerful computational and classification tool. In fact, Angular Momentum Theory proves to be essential when studying systems exhibiting rotational invariance (isotropy). In the same manner, the representation theory of $S U(1,1)$ or $S L(2, \mathbb{R})$ is useful when dealing with systems bearing conformal invariance, specially in two dimensions, where this finitedimensional symmetry can be promoted to an infinite-dimensional one (the Virasoro group). In particular, the group $S L(2, \mathbb{R})$ was used in $[2$ to define wavelets on the circle and the real line in a unified way. Furthermore, $S U(2)$ and $S U(1,1)$ CS, generalizing canonical CS of the Heisenberg-Weyl group (Gabor frames), find a great variety of applications, mainly in the study of quantum mechanical systems and their classical limit (see e.g. [3, 4, 5]). For example, ground states of superconductors and superfluids (like Bose-Einstein condensates) are coherent states. Likewise, the Lowest Landau Level (LLL) wavefunctions in Quantum Hall Effect (characterized by a quantization of the Hall conductance in two-dimensional electron systems subjected to low temperatures and strong magnetic fields) are coherent states; the formulation of such interesting

Math Subject Classifications. 32A10, 42B05, 94A12, 94A20, 81R30.

Keywords and Phrases. Holomorphic Functions, Coherent States, Discrete Fourier Transform, Sampling, Discrete Frames 
effect on the hyperboloid $S U(1,1) / U(1)$ has been recently considered in [6] (see also references therein for the extension to other geometries) and we believe that our construction of discrete frames and sampling theorems on $\mathbb{D}_{1}$ can be useful when considering numerical simulations of these systems.

Standard Continuous Wavelet Theory (see e.g. [7]) can also be seen as a chapter of CS on the group of affine transformations (translations and dilations). Here the discretization process turns out to be essential for computational applications in, for example, signal processing. These results revived interest in the question of discretization and we hope that the establishment of new sampling theorems for harmonic analysis on non-Abelian groups and their homogeneous spaces will be of importance for numerical study and simulation of those physical systems bearing that symmetries. Actually, there are some important general results about sampling and efficient computation of Fourier transforms for compact groups (see e.g. [8, 9]). However, a comprehensive study of the non-compact case is far more involved, although there is a quite well developed theory of sampling on Riemannian manifolds (see Refs. from [10] to [17]) with reconstruction formulas for bandlimited functions on homogeneous spaces. Other results in this direction have been obtained for specific groups (see e.g. [18] for a survey). For instance, we would like to point out Ref. [19] for the motion group and its engineering applications [20] (namely in robotics [21]) and [22] for discrete frames of the Poincaré group and its potential applications to Relativity Theory.

This article intends to be a further step in this direction. Completeness criteria for CS subsystems related to discrete subgroups of $S U(1,1)$ have been proved using the theory of Automorphic Forms (see e.g. [3]). Here we shall follow a different approach. Working in the open unit disk $\mathbb{D}_{1}=S U(1,1) / U(1)$ (as an appropriate realization of the Lobachevsky plane or the hyperboloid), we shall choose as sampling points for analytic functions inside $\mathbb{D}_{1}$ (carrying a unitary irreducible representation of $S U(1,1)$ of Bargmann index $s$ ) a set of $N$ equally distributed points on a circumference of radius $r<1$. For bandlimited holomorphic functions on $\mathbb{D}_{1}$ of bandlimit $M<N$ and index $s$, the resolution operator $\mathcal{A}$ is diagonal, providing a reconstruction formula by means of a (left) pseudoinverse. The Fourier coefficients can be obtained by means of the (filtered) Fourier transform of the data, allowing for a straightforward fast extension of the reconstruction algorithm. The reconstruction of arbitrary (band-unlimited) functions is not exact for a finite number $N$ of samples. However, for fast-decaying, or "quasi-bandlimited", functions it is still possible to give partial reconstruction formulas and to analyze the accuracy of the approximation in terms of $N$, the radius $r$ and the index $s$, this time through the sampled CS overlap (or reproducing kernel) $\mathcal{B}$ (see later on Sec. 2 for definitions), which exhibits a "circulant" structure and can be easily inverted using the properties of the Rectangular Fourier Matrices (RFM) and the theory of Circulant Matrices [23. This helps us to provide a reconstruction formula accomplished through an eigen-decomposition $\mathcal{B}=\mathcal{F} D \mathcal{F}^{-1}$ of $\mathcal{B}$, where $\mathcal{F}$ turns out to be the standard discrete Fourier transform matrix.

The plan of the article is as follows. In order to keep the article as self-contained as possible, we shall introduce in the next section general definitions and results about CS and frames based on a group $G$. The standard construction of CS related to the discrete series representations of $G=S U(1,1)$ is briefly sketched in Sec. 3, We refer the reader to Refs. 3, 4, 18, 24, for more information. In Section 4 we provide sampling theorems, discrete Fourier transforms and reconstruction formulas for bandlimited holomorphic functions on $\mathbb{D}_{1}$ of bandlimit $M$ and index $s$. For band-unlimited functions these reconstruction formulas are not exact for finite $N$ and we analyze the error committed in terms of $N, r$ and $s$, which tends to zero for high values of $N$. 
Finally, Sec. 5 is devoted to conclusions and outlook.

\section{Coherent States, Frames and Discretization}

Let us consider a unitary representation $U$ of a Lie group $G$ on a Hilbert space $(\mathcal{H},\langle\cdot \mid \cdot\rangle)$. Consider also the space $L^{2}(G, d g)$ of square-integrable complex functions $\Psi$ on $G$, where $d g=$ $d\left(g^{\prime} g\right), \forall g^{\prime} \in G$, stands for the left-invariant Haar measure, which defines the scalar product

$$
(\Psi \mid \Phi)=\int_{G} \bar{\Psi}(g) \Phi(g) d g .
$$

A non-zero function $\gamma \in \mathcal{H}$ is called admissible (or a fiducial vector) if $\Gamma(g) \equiv\langle U(g) \gamma \mid \gamma\rangle \in$ $L^{2}(G, d g)$, that is, if

$$
c_{\gamma}=\int_{G} \bar{\Gamma}(g) \Gamma(g) d g=\int_{G}|\langle U(g) \gamma \mid \gamma\rangle|^{2} d g<\infty
$$

A unitary representation for which admissible vector exists is called square integrable. For a square integrable representation, besides Eq. (2.2) the following property holds (see [25]):

$$
\int_{G}|\langle U(g) \gamma \mid \psi\rangle|^{2} d g<\infty, \forall \psi \in \mathcal{H}
$$

Let us assume that the representation $U$ is irreducible, and that there exists a function $\gamma$ admissible, then a system of coherent states (CS) of $\mathcal{H}$ associated to (or indexed by) $G$ is defined as the set of functions in the orbit of $\gamma$ under $G$

$$
\gamma_{g}=U(g) \gamma, \quad g \in G
$$

There are representations without admissible vectors, since the integration with respect to some subgroup diverges. In this case, or even for convenience when admissible vectors exist, we can restrict ourselves to a suitable homogeneous space $Q=G / H$, for some closed subgroup $H$. Then, the non-zero function $\gamma$ is said to be admissible $\bmod (H, \sigma)$ (with $\sigma: Q \rightarrow G$ a Borel section $*)$, and the representation $U$ square integrable $\bmod (H, \sigma)$, if the condition

$$
\int_{Q}|\langle U(\sigma(q)) \gamma \mid \psi\rangle|^{2} d q<\infty, \quad \forall \psi \in \mathcal{H}
$$

holds, where $d q$ is a measure on $Q$ "projected" from the left-invariant measure $d g$ on the whole $G$ (see [26]). Note that this more general definition of square integrability includes the previous one for $H=\{e\}$ and $\sigma$ the identity function since Eq. (2.5) reduces to Eq. (2.3), and this implies the square integrability condition (2.2).

The coherent states indexed by $Q$ are defined as $\gamma_{\sigma(q)}=U(\sigma(q)) \gamma, q \in Q$, and they form an overcomplete set in $\mathcal{H}$.

\footnotetext{
*A section $\rho: Q \rightarrow G$ of the fibre bundle $G \stackrel{H}{\rightarrow} Q$ with base $Q$ and fibre $H$ is said to be a Borel section if it is measurable with respect to the Borel $\sigma$-algebras of $Q$ and $G$.
} 
The condition (2.5) could also be written as an "expectation value"

$$
0<\int_{Q}|\langle U(\sigma(q)) \gamma \mid \psi\rangle|^{2} d q=\left\langle\psi\left|A_{\sigma}\right| \psi\right\rangle<\infty, \quad \forall \psi \in \mathcal{H}
$$

where $A_{\sigma}=\int_{Q}\left|\gamma_{\sigma(q)}\right\rangle\left\langle\gamma_{\sigma(q)}\right| d q$ is a positive, bounded, invertible operator $\dagger$

If the operator $A_{\sigma}^{-1}$ is also bounded, then the set $S_{\sigma}=\left\{\left|\gamma_{\sigma(q)}\right\rangle, q \in Q\right\}$ is called a frame (see [27] for details on frames), and a tight frame if $A_{\sigma}$ is a positive multiple of the identity, $A_{\sigma}=\lambda I, \lambda>0$.

To avoid domain problems in the following, let us assume that $\gamma$ generates a frame (i.e., that $A_{\sigma}^{-1}$ is bounded). The $C S$ map is defined as the linear map

$$
\begin{array}{rlc}
T_{\gamma}: \mathcal{H} & \longrightarrow & L^{2}(Q, d q) \\
\psi & \longmapsto & \Psi_{\gamma}(q)=\left[T_{\gamma} \psi\right](q)=\frac{\left\langle\gamma_{\sigma(q)} \mid \psi\right\rangle}{\sqrt{c_{\gamma}}} .
\end{array}
$$

Its range $L_{\gamma}^{2}(Q, d q) \equiv T_{\gamma}(\mathcal{H})$ is complete with respect to the scalar product $(\Phi \mid \Psi)_{\gamma} \equiv\left(\Phi \mid T_{\gamma} A_{\sigma}^{-1} T_{\gamma}^{-1} \Psi\right)_{Q}$ and $T_{\gamma}$ is unitary from $\mathcal{H}$ onto $L_{\gamma}^{2}(Q, d q)$. Thus, the inverse map $T_{\gamma}^{-1}$ yields the reconstruction formula

$$
\psi=T_{\gamma}^{-1} \Psi_{\gamma}=\int_{Q} \Psi_{\gamma}(q) A_{\sigma}^{-1} \gamma_{\sigma(q)} d q, \quad \Psi_{\gamma} \in L_{\gamma}^{2}(Q, d q)
$$

which expands $\psi$ in terms of CS $A_{\sigma}^{-1} \gamma_{\sigma(q)}$ with coefficients $\Psi_{\gamma}(q)=\left[T_{\gamma} \psi\right](q)$. These formulas acquire a simpler form when $A_{\sigma}$ is a multiple of the identity, as is for the case considered in this article.

When it comes to numerical calculations, the integral $A_{\sigma}=\int_{Q}\left|\gamma_{\sigma(q)}\right\rangle\left\langle\gamma_{\sigma(q)}\right| d q$ has to be discretized, which means to restrict ourself to a discrete subset $\mathcal{Q} \subset Q$. The question is whether this restriction will imply a loss of information, that is, whether the set $\mathcal{S}=\left\{\left|q_{k}\right\rangle \equiv\left|\gamma_{\sigma\left(q_{k}\right)}\right\rangle, q_{k} \in\right.$ $\mathcal{Q}\}$ constitutes a discrete frame itself, with resolution operator

$$
\mathcal{A}=\sum_{q_{k} \in \mathcal{Q}}\left|q_{k}\right\rangle\left\langle q_{k}\right|
$$

The operator $\mathcal{A}$ need not coincide with the original $A_{\sigma}$. In fact, a continuous tight frame might contain discrete non-tight frames, as happens in our case (see later on Sec. 44).

Let us assume that $\mathcal{S}$ generates a discrete frame, that is, there are two positive constants $0<b<B<\infty$ (frame bounds) such that the admissibility condition

$$
b\|\psi\|^{2} \leq \sum_{q_{k} \in \mathcal{Q}}\left|\left\langle q_{k} \mid \psi\right\rangle\right|^{2} \leq B\|\psi\|^{2}
$$

holds $\forall \psi \in \mathcal{H}$. To discuss the properties of a frame, it is convenient to define the frame (or sampling) operator $\mathcal{T}: \mathcal{H} \rightarrow \ell^{2}$ given by $\mathcal{T}(\psi)=\left\{\left\langle q_{k} \mid \psi\right\rangle, q_{k} \in \mathcal{Q}\right\}$. Then we can write $\mathcal{A}=\mathcal{T}^{*} \mathcal{T}$, and the admissibility condition (2.10) now adopts the form

$$
b I \leq \mathcal{T}^{*} \mathcal{T} \leq B I,
$$

\footnotetext{
${ }^{\dagger}$ In this paper we shall extensively use the Dirac notation in terms of "bra" and "kets" (see e.g. [2, 18]). The Dirac notation is justified by the Riesz Representation Theorem, and is valid in more general settings than Hilbert spaces of square integrable functions .
} 
where $I$ denotes the identity operator in $\mathcal{H}$. This implies that $\mathcal{A}$ is invertible. If we define the dual frame $\left\{|\tilde{q}\rangle \equiv \mathcal{A}^{-1}|q\rangle\right\}$, one can easily prove that the expansion (reconstruction formula)

$$
|\psi\rangle=\sum_{q_{k} \in \mathcal{Q}} \Psi_{k}\left|\tilde{q}_{k}\right\rangle
$$

where $\Psi_{k} \equiv\left\langle q_{k} \mid \psi\right\rangle$, converges strongly in $\mathcal{H}$, that is, the expression

$$
\mathcal{T}_{l}^{+} \mathcal{T}=\sum_{q_{k} \in \mathcal{Q}}\left|\tilde{q}_{k}\right\rangle\left\langle q_{k}\left|=\mathcal{T}^{*}\left(\mathcal{T}_{l}^{+}\right)^{*}=\sum_{q_{k} \in \mathcal{Q}}\right| q_{k}\right\rangle\left\langle\tilde{q}_{k}\right|=I
$$

provides a resolution of the identity, where $\mathcal{T}_{l}^{+} \equiv\left(\mathcal{T}^{*} \mathcal{T}\right)^{-1} \mathcal{T}^{*}$ is the (left) pseudoinverse (see, for instance, 28]) of $\mathcal{T}$ (see e.g. [24, 18] for a proof, where they introduce the dual frame operator $\tilde{\mathcal{T}}=\left(\mathcal{T}_{l}^{+}\right)^{*}$ instead $)$.

It is interesting to note that the operator $P=\mathcal{T T}_{l}^{+}$acting on $\ell^{2}$ is an orthogonal projector onto the range of $\mathcal{T}$.

From (2.12) the function $\Psi(q)$ can be obtained

$$
\Psi(q) \equiv\langle q \mid \psi\rangle=\sum_{q_{k} \in \mathcal{Q}} \Xi_{k}(q) \Psi_{k}
$$

from its samples $\Psi_{k}=\left\langle q_{k} \mid \psi\right\rangle$, through some "sinc-type" kernel

$$
\Xi_{k}(q)=\left\langle q \mid \tilde{q}_{k}\right\rangle
$$

fulfilling $\Xi_{k}\left(q_{l}\right)=P_{l k}$. A projector is obtained, instead of the identity, to account for the fact that an arbitrary set of overcomplete data $\left\{\Psi_{k}\right\} \in \ell^{2}$, can be incompatible with $|\psi\rangle \in \mathcal{H}$, and therefore they are previously projected (note that an overdetermined system of equations is being solved).

This case will be named oversampling, since there are more data than unknowns, and will be discussed in Sec. 4.1 (see also [1]). In other contexts, when eq. (2.10) holds, the set $\mathcal{Q}$ is said to be sampling for the space $\mathcal{H}[7]$.

We shall be mainly interested in cases where there are not enough points to completely reconstruct a given function $\psi$, i.e., undersampling, but a partial reconstruction is still possible. In these cases $\mathcal{S}$ does not generate a discrete frame, and the resolution operator $\mathcal{A}$ would not be invertible. But we can construct another operator from $\mathcal{T}, \mathcal{B}=\mathcal{T} \mathcal{T}^{*}$, acting on $\ell^{2}$.

The matrix elements of $\mathcal{B}$ are

$$
\mathcal{B}_{k l}=\left\langle q_{k} \mid q_{l}\right\rangle
$$

therefore $\mathcal{B}$ is the discrete reproducing kernel operator, see eq. (3.19). If the set $\mathcal{S}$ is linearly independent, the operator $\mathcal{B}$ will be invertible and a (right) pseudoinverse can be constructed for $\mathcal{T}, \mathcal{T}_{r}^{+} \equiv \mathcal{T}^{*}\left(\mathcal{T} \mathcal{T}^{*}\right)^{-1}$, in such a way that $\mathcal{T} \mathcal{T}_{r}^{+}=I_{\ell^{2}}$. As in the previous case there is another operator, $P_{\mathcal{S}}=\mathcal{T}_{r}^{+} \mathcal{T}$ acting on $\mathcal{H}$ which is an orthogonal projector onto the subspace $\mathcal{H}^{\mathcal{S}}$ spanned by $\mathcal{S}$. A pseudo-dual frame can be defined as

$$
\left|\tilde{q}_{k}\right\rangle=\sum_{q_{l} \in \mathcal{Q}}\left(\mathcal{B}^{-1}\right)_{l k}\left|q_{l}\right\rangle
$$

providing a resolution of the projector $P_{\mathcal{S}}$,

$$
\mathcal{T}_{r}^{+} \mathcal{T}=\sum_{q_{k} \in \mathcal{Q}}\left|\tilde{q}_{k}\right\rangle\left\langle q_{k}\left|=\mathcal{T}^{*}\left(\mathcal{T}_{r}^{+}\right)^{*}=\sum_{q_{k} \in \mathcal{Q}}\right| q_{k}\right\rangle\left\langle\tilde{q}_{k}\right|=P_{\mathcal{S}}
$$


Using this, a partial reconstruction (an "alias") $\hat{\psi}$ of $\psi$ is obtained,

$$
\hat{\Psi}(q)=\langle q \mid \hat{\psi}\rangle=\sum_{q_{k} \in \mathcal{Q}} L_{k}(q) \Psi_{k}
$$

from its samples $\Psi_{k}=\left\langle q_{k} \mid \psi\right\rangle$, through some "Lagrange-like" interpolating functions

$$
L_{k}(q)=\left\langle q \mid \tilde{q}_{k}\right\rangle
$$

fulfilling $L_{k}\left(q_{l}\right)=\delta_{k l}$. The alias $\hat{\psi}$ is the orthogonal projection of $\psi$ onto the subspace $\mathcal{H}^{\mathcal{S}}$, that is, $|\hat{\psi}\rangle=P_{\mathcal{S}}|\psi\rangle$. The relative (normalized) distance from the exact $\psi$ to the reconstructed function $\hat{\psi}$ is given by the relative error function:

$$
\mathcal{E}_{\psi}\left(\mathcal{H}^{\mathcal{S}}\right)=\frac{\|\psi-\hat{\psi}\|}{\|\psi\|}=\sqrt{\frac{\left\langle\psi\left|I-P_{\mathcal{S}}\right| \psi\right\rangle}{\langle\psi \mid \psi\rangle}}
$$

As mentioned above, we shall denote this case by undersampling, since there are not enough data to fully reconstruct $\psi$. In other contexts, a set $\mathcal{Q}$ is said to be interpolating if, for an arbitrary set of data $\left\{\Psi_{k}\right\}$ there exists a $|\psi\rangle \in \mathcal{H}$ such that $\left\langle q_{k} \mid \psi\right\rangle=\Psi_{k}$ [7]. This condition is satisfied in this case since $L_{k}\left(q_{l}\right)=\delta_{k l}$.

The two operators $\mathcal{A}$ and $\mathcal{B}$ are intertwined by the frame operator $\mathcal{T}, \mathcal{T} \mathcal{A}=\mathcal{B} \mathcal{T}$. If $\mathcal{T}$ were invertible, then both $\mathcal{A}$ and $\mathcal{B}$ would be invertible and $\mathcal{T}_{r}^{+}=\mathcal{T}_{l}^{+}=\mathcal{T}^{-1}$. This case would correspond to critical sampling, where both operators $\mathcal{A}$ and $\mathcal{B}$ can be used to fully reconstruct the function $\psi$. However, in many cases it is not possible to find a set of points $Q$ such that both $\mathcal{A}$ and $\mathcal{B}$ are invertible, that is, there is no critical sampling, or there are not sets $Q$ which are sampling and interpolating at the same time. The most common example is the Bargmann-Fock space of analytical functions on $\mathbb{C}$, where one can find rectangular lattices which are sampling (and therefore $\mathcal{A}$ is invertible), or which are interpolating (and thus $\mathcal{B}$ is invertible), but not both simultaneously [3, 7]. Examples of critical sampling are given by the space of band limited functions on $\mathbb{R}$ and the set $\mathbb{Z}$, which is both sampling and interpolating, and the space of functions on the Riemann sphere (or rather its stereographic projection onto the complex plane) with fixed angular momentum $s$ and the set of $N^{\text {th }}$-roots of unity, with $N=2 s+1$ [1].

It should be noted that in the case in which there is a finite number $N$ of sampling points $q_{k}$, the space $\ell^{2}$ should be substituted by $\mathbb{C}^{N}$, and the operator $\mathcal{B}$ can be identified with its matrix once a basis has been chosen.

\section{Representations of $S U(1,1)$ : Discrete Series}

Discrete series representations of $S U(1,1)$ can be found in the literature $[3$, 4 . Here we shall try to summarize what is important for our purposes, in order to keep the article as self-contained as possible.

\subsection{Coordinate Systems and Generators}

The group $S U(1,1)$ consists of all unimodular $2 \times 2$ matrices leaving invariant the pseudoEuclidean metric $\eta=\operatorname{diag}(1,-1)$ and can be parametrized as

$$
S U(1,1)=\left\{U(\zeta)=\left(\begin{array}{ll}
\zeta_{1} & \zeta_{2} \\
\bar{\zeta}_{2} & \bar{\zeta}_{1}
\end{array}\right), \zeta_{1}, \zeta_{2} \in \mathbb{C}: \operatorname{det}(U)=\left|\zeta_{1}\right|^{2}-\left|\zeta_{2}\right|^{2}=1\right\} .
$$


The group $S U(1,1)$ is locally isomorphic to the three-dimensional Lorentz group $S O(2,1)$ (the group of "rotations" of the three-dimensional pseudo-Euclidean space). More precisely $S O(2,1)=S U(1,1) / \mathbb{Z}_{2}$, where $\mathbb{Z}_{2}=\{I,-I\}$ ( $I$ is the $2 \times 2$ identity matrix $)$ is the cyclic group with two elements. The group $S U(1,1)$ acts on $\mathbb{C}$ as

$$
U(\zeta): \mathbb{C} \rightarrow \mathbb{C}, z \mapsto z^{\prime}=\frac{\zeta_{1} z+\bar{\zeta}_{2}}{\zeta_{2} z+\bar{\zeta}_{1}} .
$$

This action is not transitive, so that $\mathbb{C}$ is foliated into three orbits:

$$
\mathbb{D}_{1}=\{z \in \mathbb{C}:|z|<1\}, \quad \mathbb{C}-\overline{\mathbb{D}}_{1}=\{z \in \mathbb{C}:|z|>1\}, \mathbb{S}_{1}=\{z \in \mathbb{C}:|z|=1\} .
$$

The open unit disk $\mathbb{D}_{1}$ may be considered as the stereographical projection of the upper sheet of the two-sheet hyperboloid $\mathbb{H}^{2}=\left\{\left(x_{0}, x_{1}, x_{2}\right) \in \mathbb{R}^{3}: x_{0}^{2}-x_{1}^{2}-x_{2}^{2}=1\right\}$ onto the complex plane. The hyperboloid $\mathbb{H}^{2}$ may be identified with the set of elements of $S U(1,1)$ with $\zeta_{1}=x_{0}=$ $\cosh (\tau / 2)$ and $\zeta_{2}=x_{1}+i x_{2}=\sinh (\tau / 2) e^{i \alpha}, \tau>0, \alpha \in[0,2 \pi[$, the stereographical projection being given by $z=\zeta_{2} / \zeta_{1}=\tanh (\tau / 2) e^{i \alpha} \in \mathbb{D}_{1}$. Thus, we could also identify $\mathbb{D}_{1}$ with the coset $S U(1,1) / U(1)$, where $U(1)$ is the (diagonal) subgroup of the phase $e^{i \varphi}=\zeta_{1} /\left|\zeta_{1}\right|$.

Let us consider matrices $U(\zeta) \in S U(1,1)$ in (3.1) near the identity $I$, i.e. $\zeta \simeq e+\delta \zeta$ and $U(\zeta)=I+\sum_{a} \delta \zeta_{a} K_{a}+O\left(\delta \zeta^{2}\right)$, where $\zeta_{a}, a=0,+,-$, is a shorthand for $\zeta_{1}, \zeta_{2}, \bar{\zeta}_{2} ; e=$ $\left(\zeta_{0}, \zeta_{+}, \zeta_{-}\right)=(1,0,0)$ and the infinitesimal generators $K_{a}$ are:

$$
K_{0}=\frac{1}{2}\left(\begin{array}{cc}
1 & 0 \\
0 & -1
\end{array}\right), K_{+}=\left(\begin{array}{ll}
0 & 1 \\
0 & 0
\end{array}\right), K_{-}=\left(\begin{array}{cc}
0 & 0 \\
-1 & 0
\end{array}\right) .
$$

They close the following Lie algebra commutation relations:

$$
\left[K_{0}, K_{ \pm}\right]= \pm K_{ \pm}, \quad\left[K_{+}, K_{-}\right]=-2 K_{0} .
$$

Although we have obtained the commutators (3.5) from a particular (fundamental) representation of $S U(1,1)$ in terms of $2 \times 2$ matrices, we can abstract them and look for more general representations in terms of higher-dimensional matrices. In particular, we are interested in a class of unitary representations of $S U(1,1)$ which, being a non-compact group, must be infinitedimensional. They shall be explicitly constructed in the next section.

To finish this section, let us remind the form of the quadratic (Casimir) operator of $S U(1,1)$ :

$$
C=K_{0}^{2}-\left(K_{+} K_{-}+K_{-} K_{+}\right) / 2 .
$$

It is not difficult to verify that $C$ commutes with every $K_{a}, a=0,+,-$.

\subsection{Unitary irreducible representations: $S U(1,1)$ coherent states}

We are seeking for unitary irreducible representations of $S U(1,1)$. By Schur's lemma, for any irreducible representation of the Lie algebra $s u(1,1)$, the Casimir operator $C$ must be a multiple of the identity $I$, which we shall set $C=s(s-1) I$. Thus, an irreducible representation of $S U(1,1)$ is labelled by a single number $s$ (the Bargmann index, which we shall refer to as the symplectic spin or just "symplin"). We shall restrict ourselves to discrete series representations, which are square integrable and where $s$ is half-integer $s=1,3 / 2,2,5 / 2, \ldots$. We shall take the orthonormal basis vectors $|s, n\rangle$ in the carrier (Hilbert) space $\mathcal{H}_{s}$ to be eigenvectors of $K_{0}$ :

$$
K_{0}|s, n\rangle \equiv(n+s)|s, n\rangle .
$$


From the commutation relations (3.5), we observe that $K_{ \pm}$act as raising and lowering ladder operators, respectively, whose action on the basis vectors proves to be

$$
K_{+}|s, n\rangle=\sqrt{(n+1)(2 s+n)}|s, n+1\rangle, \quad K_{-}|s, n\rangle=\sqrt{n(2 s+n-1)}|s, n-1\rangle .
$$

Indeed, it can be easily checked that the action (3.7/3.8) preserves the commutation relations (3.5); for example:

$$
\left[K_{+}, K_{-}\right]|s, n\rangle=(n(2 s+n-1)-(n+1)(2 s+n))|s, n\rangle=-2(n+s)|s, n\rangle=-2 K_{0}|s, n\rangle
$$

and so on. From the expression (3.8) we deduce that the spectrum of $K_{0}$ is unbounded from above, that is, the Hilbert space $\mathcal{H}_{s}$ is infinite-dimensional.

Any group element $U(\zeta) \in S U(1,1)$ can also be written through the exponential map

$$
U(z, \bar{z}, \varphi)=e^{\xi K_{+}-\bar{\xi} K_{-}} e^{i \varphi K_{0}}, \quad \xi=|\xi| e^{i \beta}, z=\tanh |\xi| e^{i \beta}
$$

Note that the subgroup $U(1) \subset S U(1,1)$, generated by $K_{0}$, stabilizes any basis vector up to an overall multiplicative phase factor (a character of $U(1)$ ), i.e., $e^{i \varphi K_{0}}|s, m\rangle=e^{i(m+s) \varphi}|s, m\rangle$. Thus, according to the general prescription explained in Sec. 2. letting $Q=S U(1,1) / U(1)=\mathbb{D}_{1}$ and taking the Borel section $\sigma: Q \rightarrow G$ with $\sigma(z, \bar{z})=(z, \bar{z}, 0)$, we shall define, from now on, families of covariant coherent states $\bmod (U(1), \sigma)$ (see [18]). In simple words, we shall set $\varphi=0$ and drop it from the vectors $U(z, \bar{z}, \varphi)|s, m\rangle$.

For any choice of fiducial vector $|\gamma\rangle=|s, m\rangle$ the set of coherent states $|z, m\rangle \equiv U(z, \bar{z})|\gamma\rangle$ is overcomplete (for any $m$ ) in $\mathcal{H}_{s}$. We shall use $|\gamma\rangle=|s, 0\rangle$ as fiducial vector (i.e., the lowest weight vector), so that $K_{-}|\gamma\rangle=0$ and the coherent states

$$
|z\rangle \equiv U(z, \bar{z})|\gamma\rangle=e^{\xi K_{+}-\bar{\xi} K_{-}}|s, 0\rangle=\mathcal{N}_{s}(z, \bar{z}) e^{z K_{+}}|s, 0\rangle
$$

are holomorphic (only a function of $z$ ), apart from the normalization factor $\mathcal{N}_{s}$ which can be determined as follows. Exponentiating the relations (3.8) gives

$$
\begin{aligned}
e^{z K_{+}}|s, 0\rangle & =|s, 0\rangle+z \sqrt{2 s}|s, 1\rangle+\frac{1}{2} z^{2} \sqrt{2 s} \sqrt{2(2 s+1)}|s, 2\rangle+\ldots \\
& =\sum_{n=0}^{\infty}\left(\begin{array}{c}
2 s+n-1 \\
n
\end{array}\right)^{1 / 2} z^{n}|s, n\rangle \equiv \mathcal{N}_{s}(z, \bar{z})^{-1}|z\rangle
\end{aligned}
$$

Then, imposing unitarity, i.e., $\langle z \mid z\rangle=1$, we arrive at $\mathcal{N}_{s}(z, \bar{z})=\left(1-|z|^{2}\right)^{s}$.

The frame $\{|z\rangle, z \in \mathbb{C}\}$ is tight in $\mathcal{H}_{s}$, with resolution of unity

$$
I=\frac{2 s-1}{\pi} \int_{\mathbb{D}_{1}}|z\rangle\langle z| \frac{d^{2} z}{(1-z \bar{z})^{2}}
$$


where we denote $d^{2} z=d \operatorname{Re}(z) d \operatorname{Im}(z)$. Indeed, using (3.12) we have that

$$
\begin{aligned}
\frac{2 s-1}{\pi} \int_{\mathbb{D}_{1}}|z\rangle\langle z| \frac{d^{2} z}{(1-z \bar{z})^{2}}= & \frac{2 s-1}{\pi} \int_{\mathbb{D}_{1}} \mathcal{N}_{s}(z, \bar{z})^{2} \sum_{n, m=0}^{\infty} \sqrt{\left(\begin{array}{c}
2 s+n-1 \\
n
\end{array}\right)\left(\begin{array}{c}
2 s+m-1 \\
m
\end{array}\right)} \\
& z^{n} \bar{z}^{m}|s, n\rangle\langle s, m| \frac{d \operatorname{Re}(z) d \operatorname{Im}(z)}{(1-z \bar{z})^{2}} \\
= & 2(2 s-1) \sum_{n=0}^{\infty}\left(\begin{array}{c}
2 s+n-1 \\
n
\end{array}\right)|s, n\rangle\langle s, n| \int_{0}^{1}\left(1-r^{2}\right)^{2 s-2} r^{2 n+1} d r \\
= & \sum_{n=0}^{\infty}|s, n\rangle\langle s, n|=I,
\end{aligned}
$$

where polar coordinates were used at intermediate stage.

Using (3.12), the decomposition of the coherent state $|z\rangle$ over the orthonormal basis $\{|s, m\rangle\}$ gives the irreducible matrix coefficients

$$
\begin{aligned}
\langle z \mid s, m\rangle & =\left\langle s, 0\left|U(z, \bar{z})^{*}\right| s, m\right\rangle=\left(\begin{array}{c}
2 s+m-1 \\
m
\end{array}\right)^{1 / 2}(1-z \bar{z})^{s} \bar{z}^{m} \\
& \equiv U_{m}^{s}(z) .
\end{aligned}
$$

A general symplin $s$ function

$$
|\psi\rangle=\sum_{m=0}^{\infty} a_{m}|s, m\rangle
$$

is represented in the present complex characterization by

$$
\Psi(z) \equiv\langle z \mid \psi\rangle=\sum_{m=0}^{\infty} a_{m} U_{m}^{s}(z),
$$

which is an anti-holomorphic function of $z$ t The Fourier coefficients $a_{n}$ can be calculated through the following integral formula:

$$
a_{n}=\langle s, n \mid \psi\rangle=\frac{2 s-1}{\pi} \int_{\mathbb{D}_{1}} \Psi(z) \bar{U}_{n}^{s}(z) \frac{d^{2} z}{(1-z \bar{z})^{2}} .
$$

Note that the set of CS $\{|z\rangle\}$ is not orthogonal. The CS overlap (or Reproducing Kernel) turns out to be

$$
C\left(z, z^{\prime}\right)=\left\langle z \mid z^{\prime}\right\rangle=\frac{(1-z \bar{z})^{s}\left(1-z^{\prime} \bar{z}^{\prime}\right)^{s}}{\left(1-z^{\prime} \bar{z}\right)^{2 s}} .
$$

This quantity will be essential in our sampling procedure on the disk $\mathbb{D}_{1}$.

There are other pictures of CS for $S U(1,1)$ corresponding to other parameterizations like, for example, the one that takes $\mathbb{D}_{1}$ to the upper complex plane, but we shall not discuss them here.

\footnotetext{
${ }^{\ddagger}$ Here we abuse notation when representing the non-analytic functions $U_{m}^{s}$ and $\Psi$ simply as $U_{m}^{s}(z)$ and $\Psi(z)$, which are indeed (anti-)holomorphic up to the normalizing, non-analytic (real), pre-factor $\mathcal{N}_{s}=(1-z \bar{z})^{s}$. Usually, this pre-factor is absorbed into the integration measure in (3.13).
} 


\section{Sampling Theorem and DFT on $\mathbb{D}_{1}$}

Sampling techniques consist in the evaluation of a continuous function (a "signal") $\psi$ on a discrete set of points and later (fully or partially) recovering $\psi$ without losing essential information in the process, and the criteria to that effect are given by various forms of Sampling Theorems. Basically, the density of sampling points must be high enough to ensure the reconstruction of the function in arbitrary points with reasonable accuracy. We shall concentrate ourselves on symplin-s holomorphic functions and sample them at appropriate points.

In our case, there is a convenient way to select the sampling points in such a way that the resolution operator $\mathcal{A}$ and/or the reproducing kernel operator $\mathcal{B}$ are invertible and explicit formulas for their inverses are available. These are given by the set of $N$ points uniformly distributed on a circumference of radius $r$ :

$$
\mathcal{Q}=\left\{q_{k}=z_{k}=r e^{2 \pi i k / N}, k=0,1, \ldots N-1\right\}, r \in(0,1)
$$

which is a discrete subset of the homogeneous space $Q=S U(1,1) / U(1)=\mathbb{D}_{1}$, made of the $N^{\text {th }}$ roots of $r^{N}$, with $0<r<1 \S$. Denote by $\mathcal{S}=\left\{\left|z_{k}\right\rangle, k=0,1, \ldots, N-1\right\}$ the subset of coherent states associated with the points in $\mathcal{Q}$ and by

$$
\mathcal{H}_{s}^{\mathcal{S}} \equiv \operatorname{Span}\left(\left|z_{0}\right\rangle,\left|z_{1}\right\rangle, \ldots,\left|z_{N-1}\right\rangle\right)
$$

the subspace of $\mathcal{H}_{s}$ spanned by $\mathcal{S}$. For finite $N$ we have $\mathcal{H}_{s}^{\mathcal{S}} \neq \mathcal{H}_{s}$, so that we cannot exactly reconstruct every function $\psi \in \mathcal{H}_{s}$ from $N$ of its samples $\Psi_{k}=\left\langle z_{k} \mid \psi\right\rangle$, but we shall prove that for bandlimited functions we can always provide an exact reconstruction formula.

\subsection{Bandlimited Functions}

Definition 4.1. We define the subspace $\mathcal{H}_{s}^{M}$ of bandlimited functions of bandlimit $M<\infty$ as:

$$
\mathcal{H}_{s}^{M} \equiv \operatorname{Span}(|s, 0\rangle,|s, 1\rangle, \ldots,|s, M\rangle) .
$$

The subspace $\mathcal{H}_{s}^{M}$ is clearly a finite dimensional vector subspace of $\mathcal{H}_{s}$, but it is not invariant under the action of $S U(1,1)$. It is, however, invariant under the action of the subgroup $U(1) \subset$ $S U(1,1)$ generated by $K_{0}$ and, in this sense, it resembles the space of bandlimited functions on $\mathbb{R}$, which is invariant under the Abelian group $\mathbb{R}$.

Theorem 4.2. Given a bandlimited function $\psi \in \mathcal{H}_{s}^{M}$ on the disk $\mathbb{D}_{1}$, of band limit $M$, with a finite expansion

$$
|\psi\rangle=\sum_{m=0}^{M} a_{m}|s, m\rangle,
$$

there exists a reconstruction formula (2.12) of $\psi$

$$
\Psi(z)=\sum_{k=0}^{N-1} \Xi_{k}(z) \Psi_{k},
$$

\footnotetext{
${ }^{\S}$ In the case of the sphere 1 the sampling points were the $N^{\text {th }}$-roots of unity, which formed an abelian subgroup $\mathbb{Z}_{N}$ of $S U(2)$, and the main advantage of this was that $\mathcal{B}$ was a circulant matrix. In that case the sampling was regular [7. Now the sampling is irregular since $\mathcal{Q}$ is not a subgroup, although it is the orbit of the subgroup $\mathbb{Z}_{N}$ through the point $z=r$, and this is enough to keep the circulant structure of $\mathcal{B}$.
} 
from $N>M$ of its samples $\Psi_{k}$ taken at the sampling points in (4.1), through a "sinc-type" kernel given by

$$
\Xi_{k}(z)=\frac{1}{N}\left(\frac{1-z \bar{z}}{1-z_{k} \bar{z}_{k}}\right)^{s} \sum_{m=0}^{M}\left(\overline{z z_{k}^{-1}}\right)^{m} .
$$

Firstly, we shall introduce some notation and prove some previous lemmas.

Lemma 4.3. For $N>M$, the frame operator $\mathcal{T}: \mathcal{H}_{s}^{M} \rightarrow \mathbb{C}^{N}$ given by $\mathcal{T}(\psi)=\left\{\left\langle z_{k} \mid \psi\right\rangle, z_{k} \in \mathcal{Q}\right\}$ is such that the resolution operator $\mathcal{A}=\mathcal{T}^{*} \mathcal{T}$ is diagonal, $\mathcal{A}=\operatorname{diag}\left(\lambda_{0}, \ldots, \lambda_{M}\right)$, in the basis 4.3) of $\mathcal{H}_{s}^{M}$, with

$$
\lambda_{m} \equiv N\left(1-r^{2}\right)^{2 s}\left(\begin{array}{c}
2 s+m-1 \\
m
\end{array}\right) r^{2 m}, m=0, \ldots, M
$$

Hence, $\mathcal{A}$ is invertible in $\mathcal{H}_{s}^{M}$. Therefore, denoting $\left|\tilde{z}_{k}\right\rangle \equiv \mathcal{A}^{-1}\left|z_{k}\right\rangle$, the dual frame, the expression

$$
I_{M}=\sum_{k=0}^{N-1}\left|z_{k}\right\rangle\left\langle\tilde{z}_{k}\left|=\sum_{k=0}^{N-1}\right| \tilde{z}_{k}\right\rangle\left\langle z_{k}\right|
$$

provides a resolution of the identity in $\mathcal{H}_{s}^{M}$.

Proof. Using (3.15), the matrix elements of $\mathcal{T}$ can be written as:

$$
\mathcal{T}_{k n}=\left\langle z_{k} \mid s, n\right\rangle=\left(\begin{array}{c}
2 s+n-1 \\
n
\end{array}\right)^{1 / 2}\left(1-r^{2}\right)^{s} r^{n} e^{-2 \pi i k n / N}=\lambda_{n}^{1 / 2} \mathcal{F}_{k n},
$$

where $\mathcal{F}$ denotes the Rectangular Fourier Matrix (see [1]) given by $\mathcal{F}_{k n}=\frac{1}{\sqrt{N}} e^{-i 2 \pi k n / N}, k=$ $0, \ldots, N-1, n=0, \ldots, M$ Then, the matrix elements of the resolution operator turn out to $\mathrm{be} * *$

$$
\mathcal{A}_{n m}=\sum_{k=0}^{N-1} \mathcal{T}_{n k}^{*} \mathcal{T}_{k m}=\lambda_{n}^{1 / 2} \lambda_{m}^{1 / 2} \sum_{k=0}^{N-1} \mathcal{F}_{n k}^{*} \mathcal{F}_{k m}=\lambda_{n} \delta_{n m},
$$

where we have used the well known orthogonality relation for Rectangular Fourier Matrices (RFM) (see e.g. Appendix A of [1] for a discussion of some of their properties):

$$
\begin{aligned}
N \sum_{k=0}^{N-1} \mathcal{F}_{n k}^{*} \mathcal{F}_{k m} & =\sum_{k=0}^{N-1}\left(e^{2 \pi i(n-m) / N}\right)^{k}=\left\{\begin{array}{l}
N, \text { if }(n-m) \bmod N=0 \\
0, \text { if }(n-m) \bmod N \neq 0
\end{array}\right\} \\
& =N \delta_{(n-m) \bmod N, 0},
\end{aligned}
$$

and this equals $N \delta_{n, m}$ if $N>M$. Since $\mathcal{A}$ is diagonal with non-zero diagonal elements $\lambda_{n}$, then it is invertible and a dual frame and a (left) pseudoinverse for $\mathcal{T}$ can be constructed, $\mathcal{T}_{l}^{+} \equiv \mathcal{A}^{-1} \mathcal{T}^{*}$,

\footnotetext{
$\overline{\boldsymbol{I}}_{\text {The quantities }} \lambda_{m}$ are well defined for $m \in \mathbb{N} \cup\{0\}$ and they will be used in the case of band-unlimited functions.

"For the sake of briefness, we shall use here the same notation for Rectangular Fourier Matrices as for the square ones, namely $\mathcal{F}$, in the hope that no confusion arises (see Appendix A of 1 for a more precise distinction between both cases).

** Here and in the following we shall abuse notation denoting by $A_{i j}^{*}$ the element $\left(A^{*}\right)_{i j}=\bar{A}_{j i}$ of the hermitian conjugate matrix $A^{*}$ of $A$.
} 
providing, according to eq. (2.13), a resolution of the identity.

Proof of Theorem 4.2. From the resolution of the identity (4.8), any $\psi \in \mathcal{H}_{s}^{M}$ can be written as $|\psi\rangle=\sum_{k=0}^{N-1} \Psi_{k}\left|\tilde{z}_{k}\right\rangle$, and therefore $\Psi(z)=\langle z \mid \psi\rangle=\sum_{k=0}^{N-1} \Psi_{k}\left\langle z \mid \tilde{z}_{k}\right\rangle$. Using that $\left|\tilde{z}_{k}\right\rangle=\mathcal{A}^{-1}\left|z_{k}\right\rangle$, we derive that

$$
\begin{aligned}
\left\langle z \mid \tilde{z}_{k}\right\rangle & =\sum_{m=0}^{M}\langle z \mid s, m\rangle\left(\mathcal{A}^{-1}\right)_{m m} \mathcal{T}_{m k}^{*}=\frac{1}{\sqrt{N}} \sum_{m=0}^{M}\left(\begin{array}{c}
2 s+m-1 \\
m
\end{array}\right)^{1 / 2}(1-z \bar{z})^{s} \bar{z}^{m} \lambda_{m}^{-1 / 2} e^{2 \pi i k m / N} \\
& =\frac{1}{N}\left(\frac{1-z \bar{z}}{1-r^{2}}\right)^{s} \sum_{m=0}^{M}\left(\frac{\bar{z}}{r e^{-2 \pi i k m / N}}\right)^{m}=\Xi_{k}(z),
\end{aligned}
$$

where we have used the expression of $\langle z \mid s, m\rangle$ given by eq. (3.15).

Remark 4.4. It is interesting to note that eq. (4.5) can be interpreted as a sinc-type reconstruction formula, where the role of the sinc function is played by the function $\Xi_{k}(z)$, satisfying the "orthogonality relations" $\Xi_{k}\left(z_{l}\right)=P_{l k}$, where the operator $P=\mathcal{T}_{l}^{+}$is an orthogonal projector onto a $M$-dimensional subspace of $\mathbb{C}^{N}$, the range of $\mathcal{T}$. In the case of critical sampling, $N=M+1$, the result $\Xi_{k}\left(z_{l}\right)=\delta_{l k}$ is recovered (corresponding to an interpolation formula), but for the strict oversampling case, $N>M+1$, a projector is obtained to account for the fact that an arbitrary set of overcomplete data $\Psi_{k}, k=0, \ldots, N-1$, can be incompatible with $|\psi\rangle \in \mathcal{H}_{s}^{M}$.

A reconstruction in terms of the Fourier coefficients can be directly obtained by means of the (left) pseudoinverse of the frame operator $\mathcal{T}$ :

Corollary 4.5. (Discrete Fourier Transform) The Fourier coefficients $a_{m}$ of the expansion $|\psi\rangle=$ $\sum_{m=0}^{M} a_{m}|s, m\rangle$ of any $\psi \in \mathcal{H}_{s}^{M}$ can be determined in terms of the data $\Psi_{k}=\left\langle z_{k} \mid \psi\right\rangle$ as

$$
a_{m}=\frac{1}{\sqrt{N \lambda_{m}}} \sum_{k=0}^{N-1} e^{2 \pi i k m / N} \Psi_{k}, m=0, \ldots, M .
$$

Proof. Taking the scalar product with $\left\langle z_{k}\right|$ in the expression (4.4) of $|\psi\rangle$, we arrive at the over-determined system of equations

$$
\sum_{m=0}^{M} \mathcal{T}_{k m} a_{m}=\Psi_{k}, \quad \mathcal{T}_{k m}=\left\langle z_{k} \mid s, m\right\rangle
$$

which can be solved by left multiplying it by the (left) pseudoinverse of $\mathcal{T}, \mathcal{T}_{l}^{+}=\left(\mathcal{T}^{*} \mathcal{T}\right)^{-1} \mathcal{T}^{*}=$ $\mathcal{A}^{-1} \mathcal{T}^{*}$. Using the expressions of $\mathcal{A}^{-1}=\operatorname{diag}\left(\lambda_{0}^{-1}, \lambda_{1}^{-1}, \ldots, \lambda_{M}^{-1}\right)$, given in Lemma 4.3, and the matrix elements $\mathcal{T}_{k n}$, given by the formula (3.15), we arrive at the desired result.

Remark 4.6. Note that the Fourier coefficients $a_{m}$ are obtained as a (rectangular) discrete Fourier transform of the data $\Psi\left(z_{k}\right)$ up to a rescaling factor $1 / \sqrt{\lambda_{m}}$ which can be seen as a filter by $\mathcal{A}^{-1 / 2}$. The expression (4.13) provides a discretization of (3.18).

\subsection{Band-Unlimited Functions and Undersampling}

In the previous section we have seen that, using $N$ sampling points, we can fully reconstruct band-limited functions $\psi \in \mathcal{H}_{s}^{M}$ of band-limit up to $M=N-1$. When the reconstruction of 
a band-unlimited function $|\psi\rangle=\sum_{n=0}^{\infty} a_{n}|s, n\rangle$ from a finite number $N$ of samples is required, we cannot use the results of the previous section since the resolution operator $\mathcal{A}$ is no longer invertiblet $₫$. However, the overlapping kernel operator $\mathcal{B}$ turns out to be invertible, and a partial reconstruction can be done following the guidelines of the end of Sec. 2 (undersampling).

Contrary to the case of the sphere [1], where the Hilbert space of functions of spin $s, \mathcal{H}_{s}$, is finite-dimensional, here $\mathcal{H}_{s}$ is infinite-dimensional, and therefore in the partial reconstruction of an arbitrary function $|\psi\rangle=\sum_{n=0}^{\infty} a_{n}|s, n\rangle$ a considerable error will be committed unless further assumptions on the Fourier coefficients $a_{n}$ are made. Since $|\psi\rangle$ is normalizable, the Fourier coefficients should decrease to zero, thus even if $|\psi\rangle$ is not bandlimited, if $a_{n}$ decrease to zero fast enough, it will be "approximately" band limited if the norm of $\left|\psi_{M}^{\perp}\right\rangle \equiv \sum_{n=M+1}^{\infty} a_{n}|s, n\rangle$ is small compared to the norm of $|\psi\rangle$, for an appropriately chosen $M$. Let us formally state these ideas.

Definition 4.7. Let us define by

$$
P_{M}=\sum_{m=0}^{M}|s, m\rangle\langle s, m|
$$

the projector onto the subspace $\mathcal{H}_{s}^{M}$ of bandlimited functions of bandlimit $M$. We shall denote by

$$
\epsilon_{M+1}^{2}(\psi) \equiv \mathcal{E}_{\psi}^{2}\left(\mathcal{H}_{s}^{M}\right)=\frac{\left\langle\psi\left|I-P_{M}\right| \psi\right\rangle}{\langle\psi \mid \psi\rangle}=\frac{\sum_{n=M+1}^{\infty}\left|a_{n}\right|^{2}}{\sum_{n=0}^{\infty}\left|a_{n}\right|^{2}},
$$

the normalized squared distance [similar to (2.21)] from a band-unlimited function

$$
|\psi\rangle=\sum_{n=0}^{\infty} a_{n}|s, n\rangle \in \mathcal{H}_{s}
$$

to its orthogonal projection

$$
\left|\psi_{M}\right\rangle=P_{M}|\psi\rangle=\sum_{n=0}^{M} a_{n}|s, n\rangle
$$

onto the subspace $\mathcal{H}_{s}^{M}$. In other words, $\epsilon_{M+1}(\psi)$ is the sine of the angle between $\psi$ and $\psi_{M}$.

We hope that the (normalized) error committed when reconstructing $\psi$ from $N$ of its samples $\Psi_{k}$ will be of the order of $\epsilon_{N}(\psi)$, which will be small as long as the Fourier coefficients $a_{n}$ decay fast enough. More precisely, if $\left|a_{n}\right| \leq C / n^{\alpha}$ for some constant $C, \alpha>1 / 2$ and $n \geq N$, then

$$
\|\psi\|^{2} \epsilon_{N}^{2}(\psi)=\sum_{n=N}^{\infty}\left|a_{n}\right|^{2} \leq C^{2} \sum_{n=N}^{\infty} \frac{1}{n^{2 \alpha}} \leq C^{2} \int_{N-1}^{\infty} \frac{1}{x^{2 \alpha}} d x \leq \frac{C^{2}}{2 \alpha-1} \frac{1}{(N-1)^{2 \alpha-1}},
$$

which says that $\epsilon_{N}^{2}(\psi)=O\left(\frac{1}{N^{2 \alpha-1}}\right)$. This condition could be more formally stated by saying that $\psi$ belongs to a certain Sobolev space $\mathbb{H}^{k}$ with $k<\alpha-1 / 2$. In the next theorem we provide a partial reconstruction formula for band-unlimited functions and a bound for the error committed.

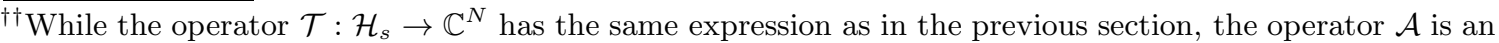
infinite dimensional matrix given by: $\mathcal{A}_{m n}=\lambda_{j+p N}^{1 / 2} \lambda_{j^{\prime}+q N}^{1 / 2} \delta_{j j^{\prime}}$, with $m=j+p N$ and $n=j^{\prime}+q N$, that is, it is a matrix made of $N \times N$ diagonal blocks.
} 
Theorem 4.8. Given a band-unlimited function $\psi \in \mathcal{H}_{s}$, there exists a partial reconstruction of $\psi$, in terms of the alias

$$
\hat{\Psi}(z)=\sum_{k=0}^{N-1} L_{k}(z) \Psi_{k},
$$

from $N$ of its samples $\Psi_{k}$, taken at the sampling points in (4.1), up to an error (2.21)

$$
\mathcal{E}_{\psi}^{2}(r, N) \equiv \mathcal{E}_{\psi}^{2}\left(\mathcal{H}_{s}^{\mathcal{S}}\right) \leq \frac{\nu_{0}(r, N)}{1+\nu_{0}(r, N)}+2 \epsilon_{N}(\psi) \sqrt{1-\epsilon_{N}^{2}(\psi)}+\epsilon_{N}^{2}(\psi) \frac{2+\nu_{0}(r, N)}{1+\nu_{0}(r, N)}
$$

with $\nu_{0}(r, N)$ given by the formula (4.31). The Lagrange-like interpolating functions (2.20) now adopt the following form:

$$
L_{k}(z)=\frac{1}{N}\left(\frac{1-z \bar{z}}{1-z_{k} \bar{z}_{k}}\right) \sum_{j=0}^{s-1} \hat{\lambda}_{j}^{-1} \sum_{q=0}^{\infty} \lambda_{j+q N}\left(\overline{z z_{k}^{-1}}\right)^{j+q N},
$$

where

$$
\hat{\lambda}_{j}=\sum_{q=0}^{\infty} \lambda_{j+q N}, j=0, \ldots, N-1,
$$

are the eigenvalues of the discrete reproducing kernel operator $\mathcal{B}=\mathcal{T}^{*}$ [defined in (2.16) with matrix elements $\left.\mathcal{B}_{k l}=\left\langle z_{k} \mid z_{l}\right\rangle\right]$ and $\lambda_{n}$ is given by [4.7), but now for $n=0,1,2, \ldots$

We shall see that the error (4.21) approaches zero when $N \rightarrow \infty$. Before tackling the proof of this theorem, we shall introduce some notation and prove some auxiliary results.

Lemma 4.9. The pseudo-frame operator $\mathcal{T}: \mathcal{H}_{s} \rightarrow \mathbb{C}^{N}$ given by $\mathcal{T}(\psi)=\left\{\left\langle z_{k} \mid \psi\right\rangle, z_{k} \in \mathcal{Q}\right\}$ [remember the construction after Eq. (2.10)] is such that the overlapping kernel operator $\mathcal{B}=$ $\mathcal{T}^{*}$ is an $N \times N$ Hermitian positive definite invertible matrix, admitting the eigen-decomposition $\mathcal{B}=\mathcal{F} \hat{D} \mathcal{F}^{*}$, where $\hat{D}=\operatorname{diag}\left(\hat{\lambda}_{0}, \ldots, \hat{\lambda}_{N-1}\right)$ is a diagonal matrix with $\hat{\lambda}_{j}$ given by 4.23) and $\mathcal{F}$ is the standard Fourier matrix.

Proof. Let us see that $\mathcal{B}$ is diagonalizable and its eigenvalues $\hat{\lambda}_{k}$ are given by the expression (4.23), which actually shows that all are strictly positive and hence $\mathcal{B}$ is invertible. This can be done by taking advantage of the circulant structure of $\mathcal{B}$ (see e.g. Appendix B in [1]). Actually, using the expression of the CS overlap (3.19), we have:

$$
\mathcal{B}_{k l}=\left\langle z_{k} \mid z_{l}\right\rangle=\left(\frac{1-r^{2}}{1-r^{2} e^{2 \pi i(l-k) / N}}\right)^{2 s} \equiv \mathcal{C}_{l-k},
$$

where the circulant structure becomes apparent. The eigenvalues of $\mathcal{B}$ are easily computed by the formula:

$$
\hat{\lambda}_{k}=\hat{D}_{k k}=\left(\mathcal{F}^{*} \mathcal{B} \mathcal{F}\right)_{k k}=\frac{1}{N} \sum_{n, m=0}^{N-1} e^{i 2 \pi k n / N} \mathcal{C}_{m-n} e^{-i 2 \pi m k / N} .
$$

If we expand the denominator of (4.24) in terms of binomial coefficients,

$$
\mathcal{C}_{l}=\left(1-r^{2}\right)^{2 s} \sum_{q=0}^{\infty}\left(\begin{array}{c}
2 s+q-1 \\
q
\end{array}\right) r^{2 q} e^{2 \pi i l q / N}
$$


insert this in (4.25) and use the general orthogonality relation for Rectangular Fourier Matrices (4.11), we arrive at (4.23). It is evident that $\hat{\lambda}_{k}>0, \forall k=0,1, \ldots, N-1$, so that $\mathcal{B}$ is invertible.

Following the general guidelines of Sec. 2, we now introduce the projector $P_{\mathcal{S}}$ :

Lemma 4.10. Under the conditions of the previous Lemma, the set $\left\{\left|\tilde{z}_{k}\right\rangle=\sum_{l=0}^{N-1}\left(\mathcal{B}^{-1}\right)_{l k}\left|z_{l}\right\rangle, k=\right.$ $0, \ldots, N-1\}$ constitutes a dual pseudo-frame for $\mathcal{S}$, the operator $P_{\mathcal{S}}=\mathcal{T}_{r}^{+} \mathcal{T}$ is an orthogonal projector onto the subspace $\mathcal{H}_{s}^{\mathcal{S}}$, where $\mathcal{T}_{r}^{+}=\mathcal{T}^{*} \mathcal{B}^{-1}$ is a (right) pseudoinverse for $\mathcal{T}$, and

$$
\sum_{k=0}^{N-1}\left|\tilde{z}_{k}\right\rangle\left\langle z_{k}\left|=\sum_{k=0}^{N-1}\right| z_{k}\right\rangle\left\langle\tilde{z}_{k}\right|=P_{\mathcal{S}}
$$

provides a resolution of the projector $P_{\mathcal{S}}$, whose matrix elements in the orthonormal base (3.7) of $\mathcal{H}_{s}$ exhibit a structure of diagonal $N \times N$ blocks:

$$
P_{m n}(r, N) \equiv\left\langle s, m\left|P_{\mathcal{S}}\right| s, n\right\rangle=\left(\lambda_{m} \lambda_{n}\right)^{1 / 2} \hat{\lambda}_{n \bmod N}^{-1} \delta_{(n-m) \bmod N, 0}, m, n=0,1,2, \ldots,
$$

with $\hat{\lambda}_{n}$ given by 4.23).

Proof. If we define $\mathcal{T}_{r}^{+}=\mathcal{T}^{*} \mathcal{B}^{-1}$ it is easy to check that $\mathcal{T} \mathcal{T}_{r}^{+}=I_{N}$ is the identity in $\mathbb{C}^{N}$. In the same way, $P_{\mathcal{S}}=\mathcal{T}_{r}^{+} \mathcal{T}$ is a projector since $P_{\mathcal{S}}^{2}=\mathcal{T}_{r}^{+} \mathcal{T} \mathcal{T}_{r}^{+} \mathcal{T}=\mathcal{T}_{r}^{+} \mathcal{T}=P_{\mathcal{S}}$ and it is orthogonal $P_{\mathcal{S}}^{*}=\left(\mathcal{T}^{*} \mathcal{B}^{-1} \mathcal{T}\right)^{*}=\mathcal{T}^{*} \mathcal{B}^{-1} \mathcal{T}=P_{\mathcal{S}}$ since $\mathcal{B}$ is self-adjoint. The resolution of the projector is provided by Eq. (2.18). Its matrix elements can be calculated through:

$$
P_{m n}(r, N)=\sum_{k, l=0}^{N-1} \mathcal{T}_{m l}^{*}\left(\mathcal{B}^{-1}\right)_{l k} \mathcal{T}_{k n}
$$

The inverse of $\mathcal{B}$ can be obtained through the eigen-decomposition:

$$
\left(\mathcal{B}^{-1}\right)_{l k}=\left(\mathcal{F} \hat{D}^{-1} \mathcal{F}^{*}\right)_{l k}=\frac{1}{N} \sum_{j=0}^{N-1} \hat{\lambda}_{j}^{-1} e^{2 \pi i j(k-l) / N}
$$

Inserting this last expression and $\mathcal{T}_{k n}=\lambda_{n}^{1 / 2} \mathcal{F}_{k n}$ in (4.29) and using the general orthogonality relation (4.11) for RFM, we finally arrive at (4.28).

The matrix elements (4.28) will be useful when computing the error function (4.21) for a general band-unlimited function (4.17). At some point, we shall be interested in their asymptotic behavior for large $N$ (large number of samples). In order to give an explicit expression of this asymptotic behavior of $P_{m n}(r, N)$, it will be useful to define the following functions:

$$
\nu_{n}(r, N) \equiv \frac{\hat{\lambda}_{n}-\lambda_{n}}{\lambda_{n}}=\sum_{u=1}^{\infty} \frac{\left(\begin{array}{c}
2 s-1+n+u N \\
n+u N
\end{array}\right)}{\left(\begin{array}{c}
2 s-1+n \\
n
\end{array}\right)} r^{2 u N}, n=0, \ldots, N-1 .
$$

In terms of $\nu_{n}(r, N)$, the matrix elements (4.28) adopt the following form:

$$
P_{m n}(r, N)=\frac{\left(\lambda_{j+p N} \lambda_{j+q N}\right)^{1 / 2}}{\sum_{u=0}^{\infty} \lambda_{j+u N}}=\frac{\left(\begin{array}{c}
2 s+j+p N-1 \\
j+p N
\end{array}\right)^{1 / 2}\left(\begin{array}{c}
2 s+j+q N-1 \\
j+q N
\end{array}\right)^{1 / 2}}{\left(_{j}^{2 s+j-1}\right)} r^{(p+q) N} \frac{1}{1+\nu_{j}(r, N)},
$$


for $m=j+p N$ and $n=j+q N$, with $j=0, \ldots, N-1$ and $p, q=0,1, \ldots$, and zero otherwise. In particular, for $n, m \leq N-1$, the projector (4.32) adopts the simple diagonal form

$$
P_{m n}(r, N)=\frac{1}{1+\nu_{n}(r, N)} \delta_{n, m}, n, m=0, \ldots, N-1 .
$$

Let us state and prove an interesting monotony property of $\nu_{n}(r, N)$.

Lemma 4.11. The functions (4.31) are strictly decreasing sequences of $n$ for $r>0$, that is:

$$
\nu_{n}(r, N)<\nu_{m}(r, N) \Leftrightarrow n>m, n, m=0, \ldots, N-1 .
$$

Proof. The quotient of binomial coefficients $\left(\begin{array}{c}2 s-1+n+u N \\ n+u N\end{array}\right) /\left(\begin{array}{c}2 s-1+n \\ n\end{array}\right)$ in (4.31) is decreasing in $n$ for any $u \in \mathbb{N}$, as can be checked by direct computation. Since this occurs for all the coefficients of the power series in (4.31), and all of them are positive, the sequence $\nu_{n}(r, N)$ is decreasing in $n$.

Now we are in conditions to prove our main theorem in this section.

Proof of Theorem 4.8; According to (2.17), the pseudo-dual frame is defined by

$$
\left|\tilde{z}_{k}\right\rangle=\sum_{l=0}^{N-1}\left(\mathcal{B}^{-1}\right)_{l k}\left|z_{l}\right\rangle=\sum_{l=0}^{N-1} \frac{1}{N} \sum_{j=0}^{N-1} \hat{\lambda}_{j}^{-1} e^{2 \pi i j(k-l) / N}\left|z_{l}\right\rangle
$$

Thus, the interpolating functions (2.20) read:

$$
L_{k}(z)=\left\langle z \mid \tilde{z}_{k}\right\rangle=\sum_{l=0}^{N-1} \frac{1}{N} \sum_{j=0}^{N-1} \hat{\lambda}_{j}^{-1} e^{2 \pi i j(k-l) / N}\left\langle z \mid z_{l}\right\rangle .
$$

Noting that

$$
\left\langle z \mid z_{l}\right\rangle=\sum_{n=0}^{\infty}\langle z \mid s, n\rangle\left\langle s, n \mid z_{l}\right\rangle=\sum_{n=0}^{\infty}\left(\begin{array}{c}
2 s+n-1 \\
n
\end{array}\right)^{1 / 2}(1-z \bar{z})^{s} \bar{z}^{n} \mathcal{T}_{l n}^{*}
$$

and using the orthogonality relation for Fourier matrices (4.11), we arrive at (4.22).

Now it remains to prove the bound (4.21) for the error. Decomposing $|\psi\rangle$ in terms of $\left|\psi_{N-1}\right\rangle \equiv P_{N-1}|\psi\rangle$ and $\left|\psi_{N-1}^{\perp}\right\rangle \equiv\left(I-P_{N-1}\right)|\psi\rangle$, we can write

$$
\begin{aligned}
\mathcal{E}_{\psi}^{2}(r, N)\|\psi\|^{2}= & \langle\psi \mid \psi\rangle-\left\langle\psi_{N-1}\left|P_{\mathcal{S}}\right| \psi_{N-1}\right\rangle-\left\langle\psi_{N-1}^{\perp}\left|P_{\mathcal{S}}\right| \psi_{N-1}^{\perp}\right\rangle \\
& -2 \operatorname{Re}\left\langle\psi_{N-1}\left|P_{\mathcal{S}}\right| \psi_{N-1}^{\perp}\right\rangle .
\end{aligned}
$$

Let us start by bounding the term

$$
\left\langle\psi_{N-1}\left|P_{\mathcal{S}}\right| \psi_{N-1}\right\rangle=\sum_{n=0}^{N-1}\left|a_{n}\right|^{2}\left\langle n\left|P_{\mathcal{S}}\right| n\right\rangle=\sum_{n=0}^{N-1}\left|a_{n}\right|^{2} \frac{1}{1+\nu_{n}(r, N)} \geq \frac{1-\epsilon_{N}^{2}(\psi)}{1+\nu_{0}(r, N)}\|\psi\|^{2},
$$

where we have used the expression (4.33), Lemma 4.11 in bounding $\frac{1}{1+\nu_{n}(r, N)} \geq \frac{1}{1+\nu_{0}(r, N)}, \forall n=$ $0, \ldots, N-1$ and the definition (4.16). Next we shall bound the term

$$
\begin{aligned}
-2 \operatorname{Re}\left\langle\psi_{N-1}\left|P_{\mathcal{S}}\right| \psi_{N-1}^{\perp}\right\rangle & \leq 2\left|\left\langle\psi_{N-1}\left|P_{\mathcal{S}}\right| \psi_{N-1}^{\perp}\right\rangle\right| \leq 2\left\|\psi_{N-1}\right\|\left\|P_{\mathcal{S}} \psi_{N-1}^{\perp}\right\| \\
& \leq 2\left\|\psi_{N-1}\right\|\left\|P_{\mathcal{S}}\right\|\left\|\psi_{N-1}^{\perp}\right\|=2 \sqrt{1-\epsilon_{N}^{2}(\psi)} \epsilon_{N}(\psi)\|\psi\|^{2}
\end{aligned}
$$


where we have used that $P_{\mathcal{S}}$ is an orthogonal projector, therefore its spectral radius is $\rho\left(P_{\mathcal{S}}\right)=$ $\left\|P_{\mathcal{S}}\right\|=1$, and the Cauchy-Schwarz inequality. Using the same arguments, we can bound the remaining term as follows:

$$
-\left\langle\psi_{N-1}^{\perp}\left|P_{\mathcal{S}}\right| \psi_{N-1}^{\perp}\right\rangle \leq\left\langle\psi_{N-1}^{\perp}\left|P_{\mathcal{S}}\right| \psi_{N-1}^{\perp}\right\rangle \leq\left\|\psi_{N-1}^{\perp}\right\|\left\|P_{\mathcal{S}}\right\|\left\|\psi_{N-1}^{\perp}\right\|=\epsilon_{N}^{2}(\psi)\|\psi\|^{2} .
$$

Putting together all this information in (4.36), we arrive at the bound (4.21) for the error.

It can be easily seen that $\lim _{N \rightarrow \infty} \nu_{0}(r, N)=0, \forall r \in(0,1)$. As a consequence, the error (4.21) goes to zero as $N \rightarrow \infty$. To obtain the order of magnitude of this error, we shall firstly give an asymptotic behavior of $\nu_{0}(r, N)$ for large $N$.

Proposition 4.12. The quantity $\nu_{0}(r, N)$ has the following asymptotic behavior (as a function of $N)$ :

$$
\nu_{0}(r, N)=\left(\begin{array}{c}
2 s-1+N \\
N
\end{array}\right) r^{2 N}+O\left(N^{2 s-1} r^{4 N}\right)
$$

as long as

$$
r<r_{0}(s, N)=\left(\frac{\left(\begin{array}{c}
2 s-1+N \\
N
\end{array}\right)}{\left(\begin{array}{c}
2 s-1+2 N \\
2 N
\end{array}\right)}\right)^{\frac{1}{2 N}}=1-\frac{(2 s-1) \ln 2}{2 N}+O\left(1 / N^{2}\right)
$$

Proof. Let us see that the first addend $(u=1)$ of

$$
\nu_{0}(r, N)=\sum_{u=1}^{\infty}\left(\begin{array}{c}
2 s-1+u N \\
u N
\end{array}\right) r^{2 u N}
$$

is dominant when $r<r_{0}(s, N)$. Indeed, the quotient between two consecutive terms of the series (4.42) is

$$
r^{2 N} \frac{\left(\begin{array}{c}
2 s-1+(u+1) N \\
(u+1) N
\end{array}\right)}{\left(\begin{array}{c}
2 s-1+u N \\
u N
\end{array}\right)}<r^{2 N} \frac{\left(\begin{array}{c}
2 s-1+2 N \\
2 N
\end{array}\right)}{\left(\begin{array}{c}
2 s-1+N \\
N
\end{array}\right)},
$$

where we have used that the quotient of binomials is decreasing in $u \in \mathbb{N}$. If we impose the terms of the series (4.42) to be monotonically decreasing for any $u \in \mathbb{N}$, i.e.

$$
r^{2 N} \frac{\left(\begin{array}{c}
2 s-1+2 N \\
2 N
\end{array}\right)}{\left(\begin{array}{c}
2 s-1+N \\
N
\end{array}\right)}<1
$$

then we arrive at (4.41). Thus, the first addend, $u=1$, of (4.42) is the leading term. Using the Stirling formula, the asymptotic behavior of the binomial $\left(\begin{array}{c}2 s-1+2 N \\ 2 N\end{array}\right)$ of the second term, $u=2$, gives the announced result (4.40).

Using the asymptotic expression (4.40), the quadratic error (4.21) approaches zero when $N \rightarrow \infty$, with the asymptotic behavior

$$
\mathcal{E}_{\psi}^{2}(r, N) \leq 2 \epsilon_{N}(\psi) \sqrt{1-\epsilon_{N}^{2}(\psi)}+2 \epsilon_{N}^{2}(\psi)+O\left(N^{2 s-1} r^{2 N}\right)
$$

for $r<r_{0}$. Thus, the reconstruction of $\psi$ by $\hat{\psi}$ is exact in this limit. 
Corollary 4.13. (Discrete Fourier Transform) The Fourier coefficients $a_{n}$ of the expansion (4.17) can be approximated by the discrete Fourier transform on the hyperboloid:

$$
\hat{a}_{n}=\frac{\lambda_{n}^{1 / 2}}{\hat{\lambda}_{n \bmod N}} \frac{1}{\sqrt{N}} \sum_{k=0}^{N-1} e^{2 \pi i n k / N} \Psi_{k}
$$

Proof. The Fourier coefficients of the alias (4.20) are given by:

$$
\hat{a}_{n}=\langle s, n \mid \hat{\psi}\rangle=\left\langle s, n\left|P_{\mathcal{S}}\right| \psi\right\rangle=\sum_{k=0}^{N-1}\left\langle s, n \mid \tilde{z}_{k}\right\rangle \Psi_{k}=\sum_{k, l=0}^{N-1}\left\langle s, n \mid z_{l}\right\rangle\left(\mathcal{B}^{-1}\right)_{k l} \Psi_{k} .
$$

Using that $\mathcal{T}_{l n}=\left\langle z_{l} \mid s, n\right\rangle=\lambda_{n}^{1 / 2} \mathcal{F}_{l n}$, given in (4.9), and the expression for the inverse of $\mathcal{B}$ given in (4.30), we obtain the final result once the orthogonality relation (4.11) for Fourier Matrices is used.

Remark 4.14. The expression for the Fourier coefficients $\hat{a}_{n}$ entails a kind of "periodization" of the original $a_{n}$. Indeed, putting

$$
\Psi_{k}=\left\langle z_{k} \mid \psi\right\rangle=\sum_{m=0}^{\infty}\left\langle z_{k} \mid m\right\rangle\langle m \mid \psi\rangle=\sum_{m=0}^{\infty} \mathcal{T}_{k m} a_{m}=\sum_{m=0}^{\infty} \lambda_{m}^{1 / 2} \mathcal{F}_{k m} a_{m}
$$

and using the orthogonality relations (4.11) we can write (4.46) as:

$$
\begin{aligned}
\hat{a}_{n} & =\frac{\lambda_{n}^{1 / 2}}{\hat{\lambda}_{n \bmod N}} \sum_{k=0}^{N-1} \mathcal{F}_{n k}^{*} \sum_{m=0}^{\infty} \lambda_{m}^{1 / 2} \mathcal{F}_{k m} a_{m} \\
& =\frac{\lambda_{n}^{1 / 2}}{\hat{\lambda}_{j}} \sum_{q=0}^{\infty} \lambda_{j+q N}^{1 / 2} a_{j+q N}, j=n \bmod N
\end{aligned}
$$

which implies

$$
\lambda_{n}^{-1 / 2} \hat{a}_{n}=\lambda_{n+p N}^{-1 / 2} \hat{a}_{n+p N} \Rightarrow \hat{a}_{n+p N}=\sqrt{\frac{\lambda_{n+p N}}{\lambda_{n}}} \hat{a}_{n}, \forall p \in \mathbb{N} .
$$

This is the "hyperbolic" counterpart of the typical aliasing effect for band-unlimited signals on the real line. $\square$

We could think that, for the case $\epsilon_{N}(\psi)=0$, we should recover the results of Section 4.1, but we shall see that this is not the case. Before, a process of truncation and filtering of $|\hat{\psi}\rangle$ in (4.20) is necessary to recover the reconstruction formula (4.5) for strict bandlimited functions (4.4). Indeed, if $M=N-1$, the truncation operation

$$
\left|\hat{\psi}_{M}\right\rangle \equiv P_{M}|\hat{\psi}\rangle=\sum_{m=0}^{M} \hat{a}_{n}|s, n\rangle
$$

followed by a rescaling (a filter) of the Fourier coefficients

$$
\left|\hat{\psi}_{M}^{R}\right\rangle \equiv R\left|\hat{\psi}_{M}\right\rangle=\sum_{m=0}^{M} \frac{\hat{\lambda}_{n}}{\lambda_{n}} \hat{a}_{n}|s, n\rangle
$$


renders the reconstruction formula for $\hat{\Psi}_{M}^{R}(z)=\left\langle z\left|R P_{M}\right| \hat{\psi}\right\rangle$ to the expression (4.5). For bandunlimited functions, the new bound for the squared normalized error turns out to be

$$
\frac{\left\|\psi-\hat{\psi}_{M}^{R}\right\|^{2}}{\|\psi\|^{2}} \leq \epsilon_{N}^{2}(\psi)+\frac{\left\langle\psi_{M}^{\perp}\left|P_{\mathcal{S}} P_{M} R^{2} P_{M} P_{\mathcal{S}}\right| \psi_{M}^{\perp}\right\rangle}{\|\psi\|^{2}} \leq \epsilon_{N}^{2}(\psi)+\epsilon_{N}^{2}(\psi)\left(1+\nu_{0}(r, N)\right)^{2},
$$

where we have followed the same steps as in the proof of Theorem 4.8, used that the spectral radius $\rho(R)=\|R\|=1+\nu_{0}(r, N)$ and that $R P_{M} P_{\mathcal{S}} P_{M}=P_{M}$. Contrary to (4.21), the new bound (4.52) is proportional to $\epsilon_{N}^{2}(\psi)$. If we, moreover, assume a behavior for $a_{n}$ as in (4.19), then we have that the error (4.52) is of order $O\left(1 / N^{2 \alpha-1}\right)$.

Let us comment on an alternative approach to the sampling of band-unlimited functions $\psi$ for small $\epsilon_{M+1}(\psi)$, which will turn out to be more convenient in a certain limit. Actually, for $\epsilon_{M+1}(\psi)<<1$ we have

$$
\left\|\psi-P_{M} \psi\right\|^{2}=\epsilon_{M+1}^{2}(\psi)\|\psi\|^{2}<<\|\psi\|^{2},
$$

so that, the reconstruction formula (4.5) for $\psi_{M}=P_{M} \psi$ would give a good approximation of $\psi$, similarly to the approach followed in [16], Section 4. The problem is that, in general, the original data $\Psi_{k}=\left\langle z_{k} \mid \psi\right\rangle$ for $\psi$ and the (unknown) "truncated" data $\Psi_{M, k}=\left\langle z_{k}\left|P_{M}\right| \psi\right\rangle$ for $\psi_{M}$ are different unless $\left\langle z_{k}\right| P_{M}=\left\langle z_{k}\right|, \forall k=0, \ldots, N-1$, which is equivalent to $\left\langle z_{k}\left|P_{M}\right| z_{k}\right\rangle=1, \forall k=$ $0, \ldots, N-1$. The following proposition studies the conditions under which such requirement is approximately satisfied.

Proposition 4.15. For large symplin $s \rightarrow \infty$ and large band limit $M \rightarrow \infty$, the diagonal matrix elements of $P_{M}$ in $\mathcal{H}_{s}^{\mathcal{S}}$ have the following asymptotic behavior:

$$
P_{M}^{s}(p) \equiv\left\langle z_{k}\left|P_{M}\right| z_{k}\right\rangle=\Theta\left(p_{c}-p\right)+O\left(\frac{1}{2 s-1+M}\right),
$$

where $p \equiv r^{2}$ with $0 \leq p<1, \Theta$ is the Heaviside function,

$$
\Theta(x)=\left\{\begin{array}{lll}
0 & \text { if } \quad x<0 \\
1 & \text { if } \quad x \geq 0
\end{array}\right.
$$

and

$$
p_{c}=\left(1+\frac{2 s-1}{M}\right)^{-1}
$$

denotes a critical squared radius. For $M>>2 s$ we have $p_{c} \lesssim 1$.

Proof. Using the expression (4.9) we have

$$
P_{M}^{s}(p) \equiv\left\langle z_{k}\left|P_{M}\right| z_{k}\right\rangle=\sum_{m=0}^{M} \mathcal{T}_{k m} \mathcal{T}_{m k}^{*}=(1-p)^{2 s} \sum_{m=0}^{M}\left(\begin{array}{c}
2 s+m-1 \\
m
\end{array}\right) p^{m}
$$

We can compute

$$
\frac{d P_{M}^{s}(p)}{d p}=-(2 s+M)\left(\begin{array}{c}
2 s+M-1 \\
M
\end{array}\right)(1-p)^{2 s-1} p^{M} .
$$

We identify here the Binomial distribution $B(2 s-1+M, p)$ (up to a factor $2 s+M$ ), which has a maximum (as a function of $p$ ) for $p_{c}=1 /\left(1+\frac{2 s-1}{M}\right)$. Using the Central Limit Theorem for $2 s-1+M \rightarrow \infty$ and the representation of the Dirac delta function as the limit of a 


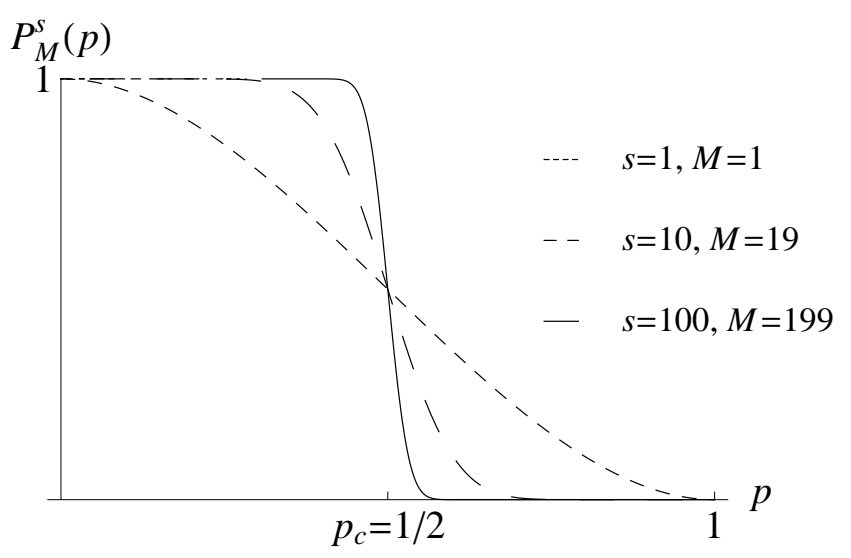

FIGURE 1: $P_{M}^{s}(p)$ as a function of $p$ for different values of $s$ and $M$, such that $p_{c}=\frac{1}{2}$.

normal distribution, we identify (4.54) as a Heaviside-type (step) function, concluding the desired result.

Figure 1 shows a plot of $P_{M}^{s}(p)$ as a function of $p$ for different values of $s$ and $M$ such that $p_{c}=1 / 2$, that is, $M=2 s-1$. It is clear how $P_{M}^{s}(p)$ approaches the step function as $M$ and $s$ grow.

Remark 4.16. The matrix elements of $P_{M}$ in $\mathcal{H}_{s}^{\mathcal{S}}$ have a circulant matrix structure. In fact, they can be seen as a Fourier transform of the coefficients $\lambda_{n}$. They have the expression $\left\langle z_{k}\left|P_{M}\right| z_{l}\right\rangle=$ $\mathcal{C}_{k-l}(p)$, where

$$
\mathcal{C}_{l}(p)=\frac{1}{N} \sum_{m=0}^{M} \lambda_{m} e^{-2 \pi i \frac{m l}{N}}
$$

Note that $\mathcal{C}_{N-l}=\mathcal{C}_{l}^{*}$, therefore the only independents elements are $\mathcal{C}_{l}, l=0, \ldots, \frac{N}{2}$. In the limit where $s$ and $M$ grow, $\left|\mathcal{C}_{l}(p)\right|$ rapidly decreases to zero when $l$ approaches $\frac{N}{2}$, as can be seen in Figure 2 ,

\section{Conclusions and Outlook}

We have proved sampling theorems and provided DFT for holomorphic functions on $\mathbb{D}_{1}$ carrying a unitary irreducible representation of $S U(1,1)$ of symplin (Bargmann index) $s$. To accomplish our objective, we used the machinery of Coherent States and discrete frames, and benefit from the theory of Circulant Matrices and Rectangular Fourier Matrices to explicitly invert resolution and reproducing kernel operators. We also paved the way for more general coset spaces $Q=G / H$ and their discretizations.

Heisenberg-Weyl (and Newton-Hooke) CS could be seen as a zero curvature limit (and large $s$ ) of $S U(2)$ (positive curvature) and $S U(1,1)$ (negative curvature) CS, a unified treatment of sampling for the three cases being possible. This is left for future work [29]. 


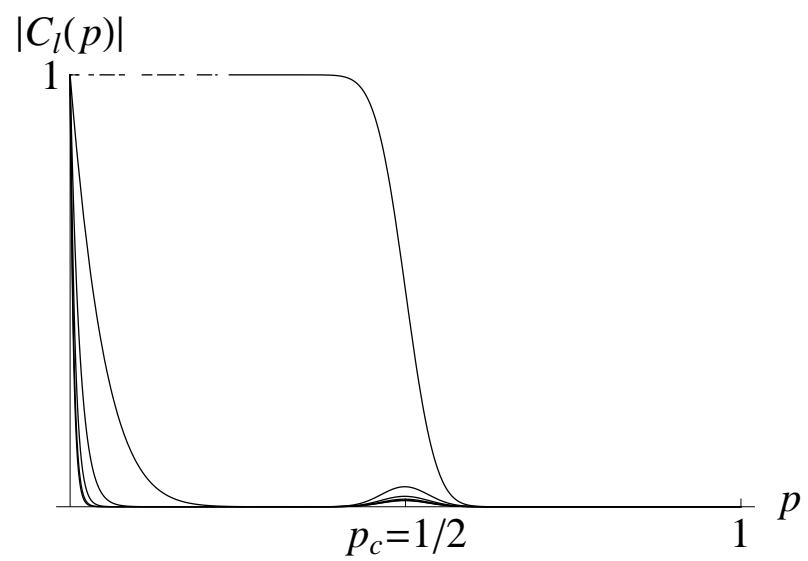

FIGURE 2: $\left|\mathcal{C}_{l}(p)\right|$ for $M=99, s=50, N=100$ and different values of $l=0,10,20,30,40,50$.

\section{Acknowledgements}

Work partially supported by the Fundación Séneca, Spanish MICINN and Junta de Andalucía under projects [03100/PI/05, 08816/PI/08, 08814/PI/08], [FIS2005-05736-C03-01, FIS2008-

06078-C03-01] and FQM219, respectively. We thank the anonymous referees for useful comments that have improved this paper and for bringing to our attention some interesting references.

\section{References}

[1] M. Calixto, J. Guerrero and J.C. Sánchez-Monreal, "Sampling Theorem and Discrete Fourier Transform on the Riemann Sphere", Journal of Fourier Analysis and Applications 14 (2008), 538-567

[2] M. Calixto and J. Guerrero, "Wavelet transform on the circle and the real line: a unified group-theoretical treatment", Applied and Computational Harmonic Analysis 21 (2006) 204-229.

[3] A. Perelomov: Generalized Coherent States and Their Applications, Springer-Verlag (1986).

[4] J.R. Klauder and Bo-Sture Skagerstam: Coherent States: Applications in Physics and Mathematical Physics, World Scientific (1985).

[5] E.P. Wigner: Group Theory and its Applications to the Quantum Mechanics of Atomic Spectra, Academic Press, New York (1959).

[6] M. Daoud and A. Jellal, "Quantum Hall Droplets on Disk and Effective Weiss-Zumino-Witten Action for Edge States", International Journal of Geometric Methods in Modern Physics 4 (2007), 1187-1204.

[7] H. Führ: Abstract Harmonic Analysis of Continuous Wavelet Transforms, Springer (2005)

[8] D. Maslen, "Efficient computation of Fourier transforms on compact groups", Journal of Fourier Analysis and Applications 4 (1998), 19-52.

[9] D. Maslen, "Sampling of functions and sections for compact Groups", Modern Signal Processing 46 (2003), 247-280.

[10] I. Pesenson, "A sampling theorem on homogeneous manifolds", Trans. Am. Math. Soc. 352 (2000), 42574269

[11] I. Pesenson, "Poincaré-type inequalities and reconstruction of Paley-Wiener functions on manifolds", J. Geom. Anal. 14 (2004), 101-121

[12] H. Feichtinger, I. Pesenson, "A reconstruction method for band-limited signals on the hyperbolic plane", Sampl. Theory Signal Image Process. 4 (2005), 107-119

[13] I. Pesenson, "Deconvolution of band limited functions on non-compact symmetric spaces", Houst. J. Math. 32 (2006), 183-204

[14] M. Ebata, M. Eguchi, S. Koizumi, K. Kumahara, "On sampling formulas on symmetric spaces", J. Fourier 
Anal. Appl. 12 (2006), 1-15

[15] M. Ebata, M. Eguchi, S. Koizumi, K. Kumahara, "Analogues of sampling theorems for some homogeneous spaces", Hiroshima Math. J. 36 (2006), 125-140

[16] I. Pesenson, "Paley-Wiener Approximations and Multiscale Approximations in Sobolev and Besov Spaces on Manifolds", J. Geom. Anal. 19 (2009), 390-419

[17] M. B. Stenzel, "A Reconstruction Theorem for Riemannian Symmetric Spaces of Noncompact Type", J. Fourier Anal. Appl. 15 (2009), 839-856

[18] S.T. Ali, J-P. Antoine, J.P. Gazeau: Coherent States, Wavelets and Their Generalizations, Springer (2000)

[19] A. Kyatkin and G.S. Chirikjian, "Algorithms for fast convolutions on motion groups", Applied and Computational Harmonic Analysis 9 (2000), 220-241

[20] G.S. Chirikjian and A. Kyatkin, "Engineering applications of noncommutative harmonic analysis", CRC Press (2001).

[21] A. Kyatkin and G.S. Chirikjian, "Computation of robot configuration and workspaces via the Fourier transform on the discrete motion-group", International Journal of Robotics Research 18 (1999), 601-615

[22] J-P. Antoine and A.L. Hohouto, "Discrete frames of Poincaré coherent states in $1+3$ dimensions", Journal of Fourier Analysis and Applications 9 (2003), 141-173

[23] P.J. Davis: Circulant Matrices, Chelsea Publishing, NY (1994)

[24] M. Holschneider: Wavelets: an analysis tool, Oxford University Press (1998)

[25] A. Grossmann, J. Morlet and T. Paul, "Transforms associated to square integrable group representations I. General results", J. Math. Phys. 26 (1985) 2473-2479.

[26] J. Guerrero and V. Aldaya, "Invariant Measures on Polarized submanifolds in Group Quantization", J. Math. Phys. 41 (2000), 6747-6765

[27] O. Christensen: An introduction to Frames and Riesz Bases, Birkhäuser, Biston (2003)

[28] A. Ben-Israel, T.N.E. Greville: Generalized Inverses, Springer-Verlag (2003)

[29] M. Calixto, J. Guerrero and J.C. Sánchez-Monreal, "Sampling Theorems and Discrete Fourier Transforms on Curved Phase Spaces of Constant Curvature: a Unified Treatment", in progress.

Departamento de Matemática Aplicada y Estadística, Universidad Politécnica de Cartagena

Paseo Alfonso XIII 56, 30203 Cartagena, Spain

e-mail: Manuel.Calixto@upct.es

Departamento de Matemática Aplicada, Universidad de Murcia, Facultad de Informática

Campus de Espinardo, 30100 Murcia, Spain

e-mail: juguerre@um.es

Departamento de Matemática Aplicada y Estadística, Universidad Politécnica de Cartagena

Paseo Alfonso XIII 56, 30203 Cartagena, Spain

e-mail: JCarlos.Sanchez@upct.es 